\title{
Seebeck Coefficient in a Cuprate Superconductor: Particle-Hole Asymmetry in the Strange Metal Phase and Fermi Surface Transformation in the Pseudogap Phase
}

\author{
A. Gourgout, ${ }^{1, *}$ G. Grissonnanche,${ }^{1,2,3, *, \dagger}$ F. Laliberté, ${ }^{1}$ A. Ataei,${ }^{1}$ L. Chen $\odot,{ }^{1}$ S. Verret, ${ }^{1}$ \\ J.-S. Zhou $\odot,{ }^{4}$ J. Mravlje, ${ }^{5}$ A. Georges, ${ }^{6,7,8,9}$ N. Doiron-Leyraud, ${ }^{1}$ and Louis Taillefer ${ }^{1,10, \$}$ \\ ${ }^{1}$ Institut Quantique, Département de physique and RQMP, Université de Sherbrooke, \\ Sherbrooke, Québec, Canada \\ ${ }^{2}$ Laboratory of Atomic and Solid State Physics, Cornell University, Ithaca, New York, USA \\ ${ }^{3}$ Kavli Institute at Cornell for Nanoscale Science, Ithaca, New York, USA \\ ${ }^{4}$ Materials Science and Engineering Program/Mechanical Engineering, \\ University of Texas-Austin, Austin, Texas, USA \\ ${ }^{5}$ Department of Theoretical Physics, Institute Jožef Stefan, Ljubljana, Slovenia \\ ${ }^{6}$ Collège de France, 11 place Marcelin Berthelot, Paris, France \\ ${ }^{7}$ Center for Computational Quantum Physics, Flatiron Institute, New York, New York, USA \\ ${ }^{8}$ CPHT, CNRS, Ecole Polytechnique, IP Paris, Palaiseau, France \\ ${ }^{9} D Q M P$, Université de Genève, Geneva, Switzerland \\ ${ }^{10}$ Canadian Institute for Advanced Research, Toronto, Ontario, Canada
}

(Received 10 June 2021; revised 7 December 2021; accepted 4 January 2022; published 25 February 2022)

\begin{abstract}
We report measurements of the Seebeck effect in both the $a b$ plane $\left(S_{a}\right)$ and along the $c$ axis $\left(S_{c}\right)$ of the cuprate superconductor $\mathrm{La}_{1.6-x} \mathrm{Nd}_{0.4} \mathrm{Sr}_{x} \mathrm{CuO}_{4}$ (Nd-LSCO), performed in magnetic fields large enough to suppress superconductivity down to low temperature. We use the Seebeck coefficient as a probe of the particle-hole asymmetry of the electronic structure across the pseudogap critical doping $p^{\star}=0.23$. Outside the pseudogap phase, at $p=0.24>p^{\star}$, we observe a positive and essentially isotropic Seebeck coefficient as $T \rightarrow 0$. That $S>0$ at $p=0.24$ is at odds with expectations given the electronic band structure of Nd-LSCO above $p^{\star}$ and its known electronlike Fermi surface. We can reconcile this observation by invoking an energy-dependent scattering rate with a particle-hole asymmetry, possibly rooted in the non-Fermi-liquid nature of cuprates just above $p^{\star}$. Inside the pseudogap phase, for $p<p^{\star}, S_{a}$ is seen to rise at low temperature as previously reported, consistent with the drop in carrier density $n$ from $n \simeq 1+p$ to $n \simeq p$ across $p^{\star}$ as inferred from other transport properties. In stark contrast, $S_{c}$ at low temperature becomes negative below $p^{\star}$, a novel signature of the pseudogap phase. The sudden drop in $S_{c}$ reveals a change in the electronic structure of Nd-LSCO upon crossing $p^{\star}$. We can exclude a profound change of the scattering across $p^{\star}$ and conclude that the change in the out-of-plane Seebeck coefficient originates from a transformation of the Fermi surface.
\end{abstract}

Subject Areas: Condensed Matter Physics

\section{INTRODUCTION}

The pseudogap phase of cuprate superconductors, in particular, its link with high-temperature superconductivity, remains an enduring mystery of condensed matter physics. While no clear phase transition at its characteristic temperature $T^{\star}$ is observed in transport and

\footnotetext{
*These authors contributed equally to this work. †gael.grissonnanche@cornell.edu

*louis.taillefer@usherbrooke.ca
}

Published by the American Physical Society under the terms of the Creative Commons Attribution 4.0 International license. Further distribution of this work must maintain attribution to the author(s) and the published article's title, journal citation, and DOI. thermodynamic properties, the low-temperature crossing of its critical doping $p^{\star}$ in the absence of superconductivity yields clear signatures of a transition [1]. The normal-state Hall coefficient of $\mathrm{YBa}_{2} \mathrm{Cu}_{3} \mathrm{O}_{y}$ (YBCO) [2], $\mathrm{La}_{1.6-x} \mathrm{Nd}_{0.4} \mathrm{Sr}_{x} \mathrm{CuO}_{4}$ (Nd-LSCO) [3], and $\mathrm{Bi}_{2} \mathrm{Sr}_{2-x} \mathrm{La}_{x} \mathrm{CuO}_{6+\delta}$ (Bi2201) [4] shows that the carrier density $n$ changes abruptly from $n \simeq 1+p$ to $n \simeq p$ when crossing $p^{\star}$ from above. This is also observed in the electrical resistivity of $\mathrm{La}_{2-x} \mathrm{Sr}_{x} \mathrm{CuO}_{4}$ (LSCO) [5] and Nd-LSCO [3] and the thermal conductivity (at $T \rightarrow 0$ ) of Nd-LSCO [6]. Clear evidence for a transformation of the Fermi surface of Nd-LSCO across $p^{\star}$ was recently obtained from angle-dependent magnetoresistance (ADMR) [7]. The low-temperature electronic specific heat $[8,9]$ shows a logarithmic divergence at $p^{\star}$, evidence for a quantum phase transition. 
Recently, the thermopower was used as a complementary probe of the carrier density at low temperatures across $p^{\star}$ in Nd-LSCO [10]. Indeed, in the $T=0$ limit and for a single parabolic band, the Seebeck coefficient depends on only two parameters: the coefficient of electronic specific heat $\gamma=C_{\mathrm{el}} / T$ and the carrier density $n$ ( $e$ is the electron charge):

$$
\frac{S}{T}=\frac{\gamma}{n e} .
$$

According to this expression, the ratio $S / T$ can therefore be seen as the specific heat per carrier. Simplified as it is, this physical picture is shown by Behnia, Jaccard, and Flouquet [11] to account empirically for the observed low-temperature value of the Seebeck coefficient for a great variety of materials that includes common metals and several strongly correlated materials.

In Nd-LSCO, Eq. (1) articulates that the sudden increase in the in-plane Seebeck coefficient $S_{a} / T$ as $p$ crosses below $p^{\star}[10]$ is consistent with the drop in $n$ at $p^{\star}$ inferred from the Hall effect, electrical resistivity, and thermal conductivity measurements. Although the relative change in $S_{a} / T$ across $p^{\star}$ seems well captured by Eq. (1), it is unable to explain the positive sign of the in-plane Seebeck coefficient, which is at odds with the electronlike band of NdLSCO above $p^{\star}$ [12]. To date, the sign of the Seebeck coefficient in overdoped cuprates remains a mystery.

At a more fundamental level, the Seebeck coefficient is actually a ratio of transport coefficients which involves states immediately below and above the Fermi level, in contrast with electrical transport that is sensitive only to the properties of the Fermi surface. As a result, the Seebeck coefficient is controlled by the particle-hole asymmetry between occupied and unoccupied states around the Fermi level $[13,14]$. This asymmetry can originate both from the dispersion of electronic excitations (band structure) and from the energy dependence of the scattering rate. Accounting for these effects clearly goes beyond the simplified expression Eq. (1).

In the present article, we present measurements of the out-of-plane Seebeck coefficient $S_{c}$. We report a sudden qualitative change of $S_{c}$ in Nd-LSCO across $p^{\star}$. We show that, in order to successfully describe the behavior of the Seebeck coefficient along both directions, it is crucial to take into account the particle-hole asymmetry ("skewness") in the energy dependence of the scattering rate.

Nd-LSCO is a single-layer, tetragonal cuprate superconductor with low critical temperature $T_{c}$ and field $H_{c 2}$, making it an ideal candidate to study the field-induced normal state down to low temperatures. Its phase diagram is shown in Fig. 1, where the pseudogap temperature $T^{\star}$ extracted from resistivity measurements [10] is displayed and seen to be in agreement with angle-resolved photoemission spectroscopy (ARPES) measurements [15].

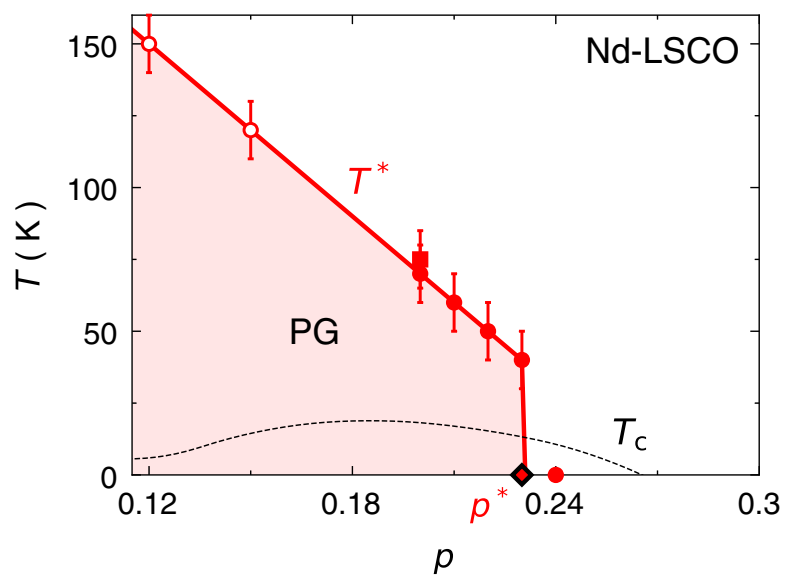

FIG. 1. Temperature-doping phase diagram of the cuprate superconductor Nd-LSCO. Red points represent the temperature below which the pseudogap phase appears, $T^{\star}$, obtained from resistivity $[3,16]$ (full points show the dopings studied here) and ARPES [15] (full square) measurements. The red shaded area represents the pseudogap (PG) phase, which disappears at the critical doping $p^{\star}=0.23 \pm 0.01$ (red diamond). The full line is a guide to the eye. The superconducting phase (in zero field) is bounded by $T_{c}$ (dashed line).

Above $p^{\star}$, at $p=0.24$, our measurements reveal that $S_{a}$ and $S_{c}$ are positive and essentially equal at low temperature, both ending with a $\log (1 / T)$ dependence below about $10 \mathrm{~K}$. Although it is consistent with Eq. (1), the isotropy of the Seebeck coefficient at $p=0.24$ as $T \rightarrow 0$ cannot be understood in terms of the band structure, since it predicts the wrong magnitude of $S_{c}$ and is of the wrong sign for $S_{a}$. We show that taking into account the angle dependence and temperature dependence of the scattering rate deduced from ADMR [17] and adding a linear energy dependence that is particle-hole asymmetric reconciles the calculated and measured Seebeck coefficients along both directions.

Upon crossing into the pseudogap phase at $p^{\star}$, we find that $S_{c}$ becomes negative at low temperature, in contrast to $S_{a}$, which remains positive. This contrasts with Eq. (1) that predicts that the drop in $n$ at $p^{\star}$ should also be observed in the interlayer $c$ axis component $S_{c}$. The negative $S_{c}$ reflects a profound transformation of the electronic structure across $p^{\star}$.

\section{METHODS}

Single crystals of Nd-LSCO are grown by a traveling solvent or floating zone technique in an image furnace, with a Nd content of 0.4 and doping $p=0.20,0.21,0.22,0.23$, and 0.24 (for more details, see Collignon et al. [3]). These are subsequently cut into bar-shaped samples with typical dimensions of $1 \mathrm{~mm} \times 0.5 \mathrm{~mm} \times 0.2 \mathrm{~mm}$. For each doping, separate samples with their length, respectively, along the $a$ axis and the $c$ axis are cut.

We measure the Seebeck coefficient using an ac technique (alternating current) that was originally developed for 
thin films [18] and that we have adapted for measuring bulk materials. An ac thermal excitation is generated through the sample by sending an electric current at a frequency $\omega \sim$ $1 \mathrm{~Hz}$ to a $5 \mathrm{k} \Omega$ strain gauge used as a heater. This generates a longitudinal thermal gradient $\Delta T_{\mathrm{ac}}$ along the length of the sample, measured at a frequency $2 \omega$ using two absolute type $\mathrm{E}$ thermocouples. In response to that thermal gradient, a Seebeck voltage $\Delta V_{\text {ac }}$ is measured at a frequency $2 \omega$ with phosphor-bronze wires using the same contacts as for $\Delta T_{\text {ac }}$, which eliminates uncertainties associated with the geometric factor. The Seebeck coefficient is then given by $S=-\Delta V_{\mathrm{ac}} / \Delta T_{\mathrm{ac}}$. Our method differs from previous ac Seebeck measurements by accounting for both the modulus and the phase of the thermal and thermoelectric signals. This technique can be, in principle, extended to all orders in frequencies. The advantages of using an ac method over dc (direct current) is that the dc technique requires one to measure the Seebeck coefficient at constant temperature steps and wait the necessary time to reach equilibrium, which can take minutes and even hours closer to room temperature; it is also limited in resolution to few-millikelvin fluctuations for the thermal gradient and few nanovolts for the Seebeck voltage. The ac Seebeck technique, on the other hand, does not require for the sample to reach equilibrium, which makes the measurements quasi-instantaneous, and comes down to a resolution in the approximately $10-\mu \mathrm{K}$ range because of the use of lock-in amplifiers. However, it takes a real time measurement of the sample temperature, which comes with its own set of challenges.

The thermocouple and Seebeck voltages are amplified using EM Electronics A10 preamplifiers and picked up using SR830 lock-in amplifiers at the thermal excitation frequency $2 \omega$. Our method allows us to measure $S(T)$ continuously from 2 to $300 \mathrm{~K}$ within a few hours and with a much better signal-to-noise ratio compared to a standard steady-state dc technique. It is carefully benchmarked against a steady-state dc method on several samples.

\section{RESULTS}

\section{A. Outside the pseudogap phase $\boldsymbol{p}>\boldsymbol{p}^{\star}$}

In Fig. 2(a), we show $S_{a} / T$ and $S_{c} / T$ as a function of the temperature in Nd-LSCO just outside the pseudogap phase, at $p=0.24$, in the normal state induced by an applied field of $H=16 \mathrm{~T}\left(H_{c 2}=9 \mathrm{~T}\right.$ in Nd-LSCO and Eu-LSCO at $p=0.24$ [8]). We observe that $S_{a}$ is negative at room temperature, similar to what is observed in overdoped Bi2201 [14], for example. Upon cooling, $S_{a} / T$ increases and changes sign around $250 \mathrm{~K}$, reaching a positive value of about $0.45 \mu \mathrm{V} / \mathrm{K}^{2}$ at the lowest measured temperature $T \simeq 2 \mathrm{~K}$. Conversely, $S_{c} / T$ starts from a positive value at $300 \mathrm{~K}$, and it increases until it reaches a plateau around $60 \mathrm{~K}$ but at lower temperature starts to increase again. Below about $10 \mathrm{~K}$, a remarkable isotropy appears: $S_{a} / T$
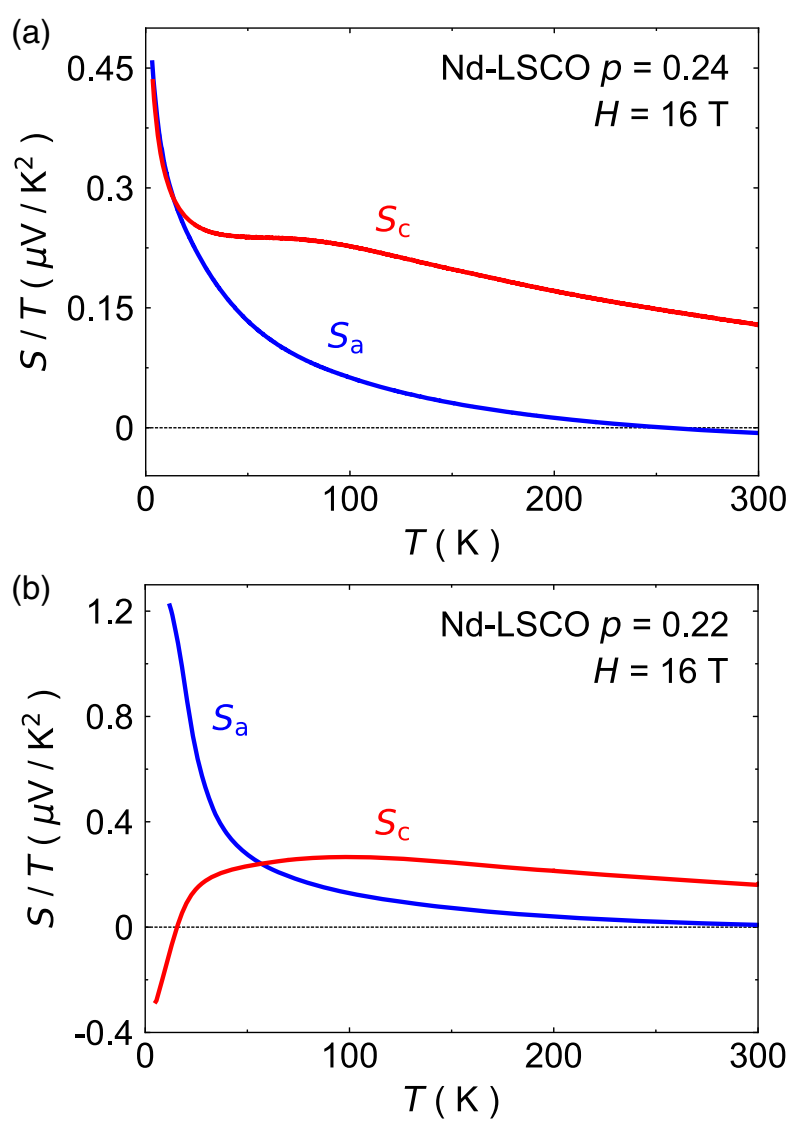

FIG. 2. In-plane $\left(S_{a}\right.$, blue) and out-of-plane $\left(S_{c}\right.$, red) Seebeck coefficient of Nd-LSCO, plotted as $S / T$ vs $T$ at $H=16 \mathrm{~T}$ for (a) $p=0.24>p^{\star}$ and (b) $p=0.22<p^{\star}$.

and $S_{c} / T$ are essentially identical despite the quasitwo-dimensional character of the electronic structure and associated anisotropy in the electrical resistivity (in

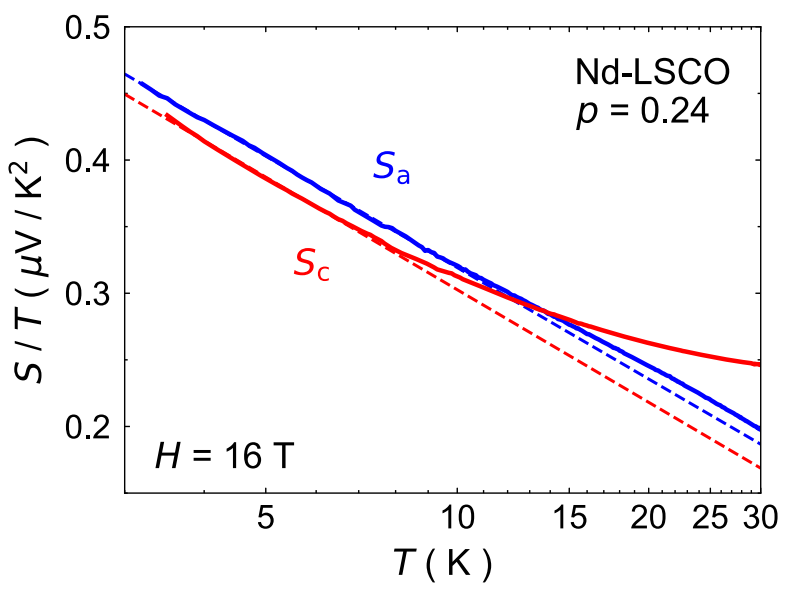

FIG. 3. In-plane $\left(S_{a}\right.$, blue) and out-of-plane $\left(S_{c}\right.$, red) Seebeck coefficients of Nd-LSCO at $p=0.24>p^{\star}$ plotted as $S / T$ vs $\log (T)$ at $H=16 \mathrm{~T}$. Note the logarithmic dependence below approximately $10 \mathrm{~K}$, where $S$ is essentially isotropic $\left(S_{a} \simeq S_{c}\right)$. 
Nd-LSCO at $p=0.24$, the $c$-axis resistivity $\rho_{c}$ is approximately 250 times larger than $a$-axis resistivity $\left.\rho_{a}[16]\right)$.

As seen in Fig. 3, zooming in on the low-temperature data reveals that $S_{a} / T$ and $S_{c} / T$ both display a $\log (1 / T)$ dependence below approximately $10 \mathrm{~K}$, as previously reported for $S_{a} / T$ in Nd-LSCO [19], Eu-LSCO [20] at $p=0.24$, and Bi2201 near $p^{\star}$ [4]. This behavior is also observed in the electronic specific heat of Nd-LSCO and Eu-LSCO at $p=0.24$, whereby $C_{\mathrm{el}} / T \sim \log (1 / T)$ below approximately $10 \mathrm{~K}$ is interpreted as a signature of quantum criticality in the vicinity of the pseudogap critical point $[8,9]$. Another typical signature of quantum criticality is a $T$-linear dependence of the electrical resistivity as $T \rightarrow 0$ observed in Nd-LSCO at $p=0.24$ both in the plane and along the $c$ axis [16].

\section{B. Inside the pseudogap phase $\boldsymbol{p}<\boldsymbol{p}^{\star}$}

Now going inside the pseudogap phase, we see in Fig. 4(a) that $S_{a} / T$ undergoes a large enhancement at low temperature for $p<0.23$. This was previously reported and discussed in detail in Ref. [10], and here we show the continuous $T$-dependent curves of $S_{a} / T$ in $H=16 \mathrm{~T}$ obtained using our ac technique (our ac data are in excellent agreement with
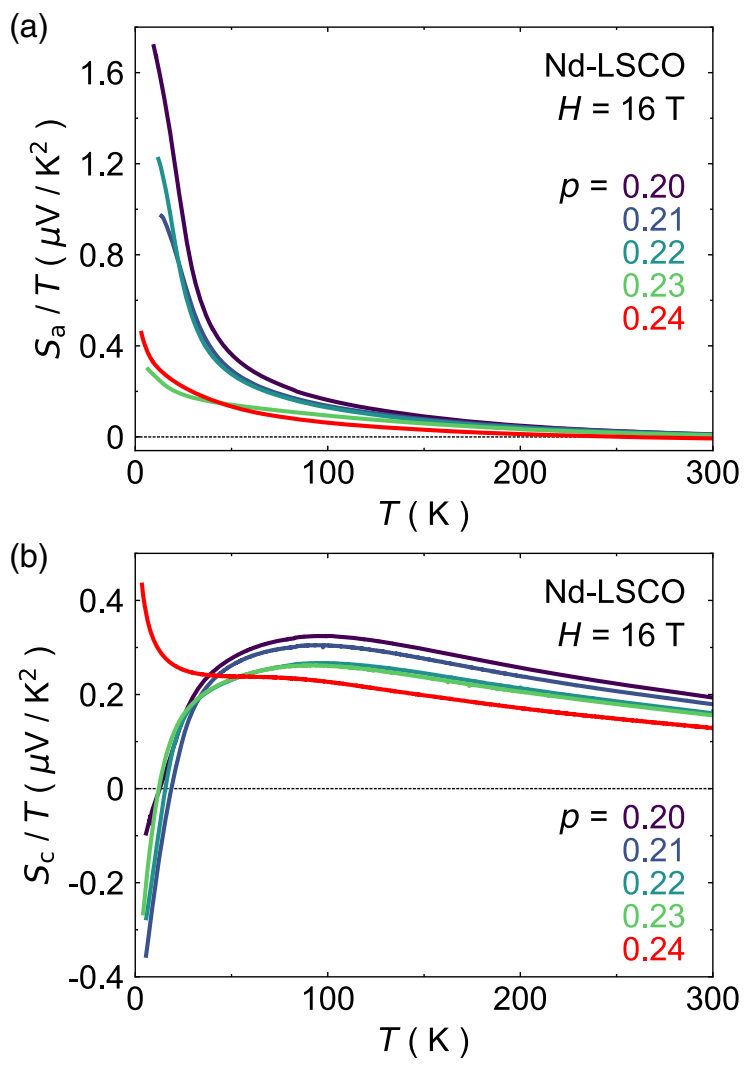

FIG. 4. (a) $S_{a} / T$ and (b) $S_{c} / T$ as a function of the temperature, at $H=16 \mathrm{~T}$, in Nd-LSCO at dopings as indicated, on both sides of the pseudogap critical point $p^{\star}=0.23$. Note the qualitative change in $S_{c}$ upon crossing below $p^{\star}$, whereby $S_{c}$ suddenly becomes negative at low $T$. the previous dc data [10]). As seen in the Hall effect and electrical resistivity of Nd-LSCO [3], the enhancement of $S_{a} / T$ occurs below $T^{\star}$ and reaches a maximum as $T \rightarrow 0$ that corresponds roughly to a fivefold increase between $p=0.24$ and $p=0.20$. In agreement with Eq. (1), this roughly matches the change in carrier density from $n \simeq 1+p=$ 1.24 at $p=0.24$ to $n \simeq p=0.20$ at $p=0.20$, suggesting that it is the principal cause for the enhancement of these three transport coefficients $\left(R_{H}, \rho\right.$, and $\left.S\right)$ inside the pseudogap phase.

Turning to $S_{c} / T$ below $p^{\star}$, at $p=0.22$, we see in Fig. 2 that it tracks $S_{c} / T$ at $p=0.24$ down to about $100 \mathrm{~K}$ but then drops upon further cooling to reach negative values below $T \sim 20 \mathrm{~K}$. This behavior is seen for all the measured dopings at and below $p^{\star}$ [Fig. 4(b)], showing that it is a property of the pseudogap phase. [We stress that the field dependence of $S_{c}$ is very weak, as seen in Fig. 5(b), where the curves taken at $H=0$ and $16 \mathrm{~T}$ essentially overlap, establishing that the sole effect of the field is to suppress superconductivity and reveal $S_{c} / T$ at low temperatures, not to induce the negative $S_{c} / T$.]

As we discuss below, the highly contrasting behavior between $S_{a} / T$ and $S_{c} / T$ below $p^{\star}$ contradicts Eq. (1), that
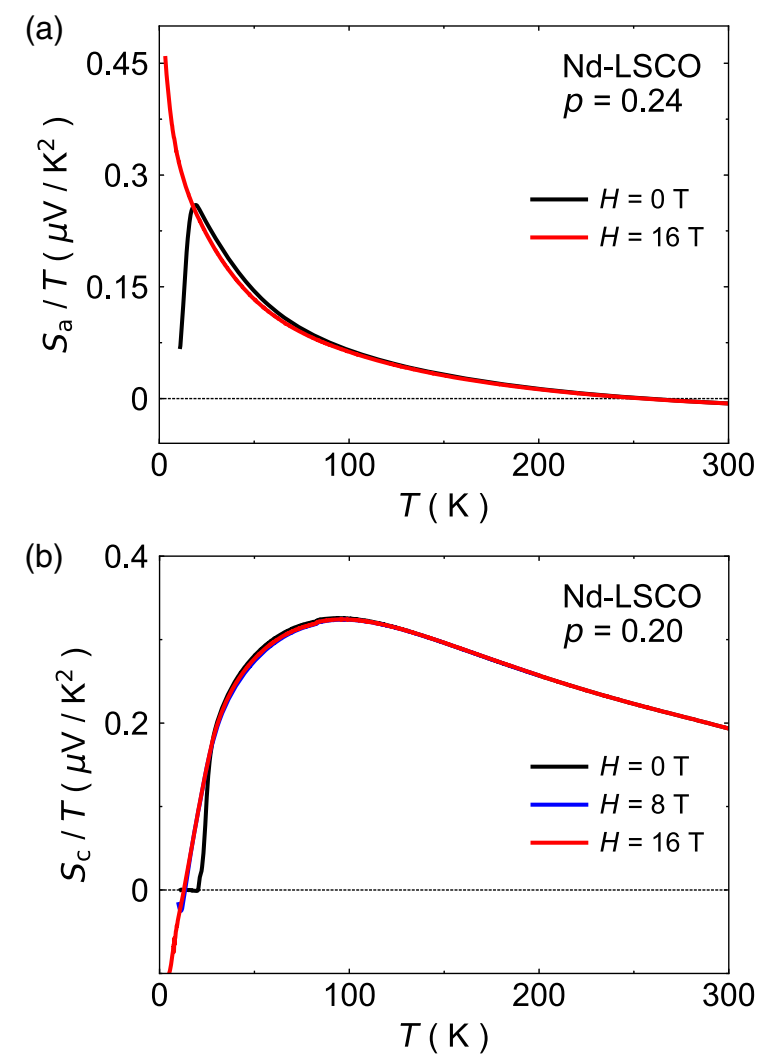

FIG. 5. $S / T$ of Nd-LSCO as a function of the temperature for different fields applied parallel to the $c$ axis. (a) $S_{a} / T$ at $p=0.24$ in $H=0$ and $16 \mathrm{~T}$. (b) $S_{c} / T$ at $p=0.20$ in $H=0,8$, and $16 \mathrm{~T}$. We see that applying a field has very little effect on the normalstate $S_{c} / T$ curve, except for suppressing superconductivity and revealing it down to low temperatures. 
predicts an isotropy between the two current directions, and is a puzzle for standard theories of the Seebeck effect, likely carrying significant hints for the underlying nature of the pseudogap phase. Whatever mechanism causes the drop in carrier density from $n=1+p$ to $n=p$ at $p^{\star}$ and the associated Fermi surface transformation observed by ADMR measurements [7], it seems to also affect the asymmetry of the band dispersion around the Fermi level in an anisotropic fashion, as attested by the negative $S_{c} / T$. $S_{c}$, therefore, adds to the list of transport properties undergoing a dramatic change upon crossing $p^{\star}$, such as the negative thermal Hall effect [21], attributed to chiral phonons [22], that suddenly appears in the pseudogap phase of Nd-LSCO.

\section{DISCUSSION}

\section{A. $p>p^{\star}$ : Particle-hole asymmetric energy-dependent scattering rate}

Previous studies of the Seebeck coefficient of cuprates largely focus on the behavior above $T_{c}$, revealing that the inplane $S(T)$ decreases with increasing doping [23] and is negative at high temperature and high doping, as seen, for instance, in $\mathrm{Bi}_{2} \mathrm{Sr}_{2} \mathrm{CaCu}_{2} \mathrm{O}_{8+x}$ (Bi2212) [24], LSCO [25], Bi2201 [14], and $\mathrm{HgBa}_{2} \mathrm{CuO}_{4+\delta}$ [26]. Nd-LSCO follows this trend as a function of the doping at $T=300 \mathrm{~K}$, as shown in Fig. 6, and as a function of the temperature in Fig. 2(a). In Nd-LSCO above $p^{\star}$, at $p=0.24, S_{a}(T)$ is slightly negative at $300 \mathrm{~K}$ but becomes positive below about $T \sim 250 \mathrm{~K}$ and keeps increasing upon further cooling [Fig. 2(a)]. This behavior contrasts with overdoped Bi2201, where $S_{a}(T)$ is negative at all temperatures at dopings close to $p^{\star}$ [4].

In order to understand the observed behavior of $S_{a} / T$ and $S_{c} / T$, we turn to Boltzmann transport theory in a relaxation time approximation, using the experimentally determined (quasiparticle) band dispersion $E(\vec{k})$ of Nd-

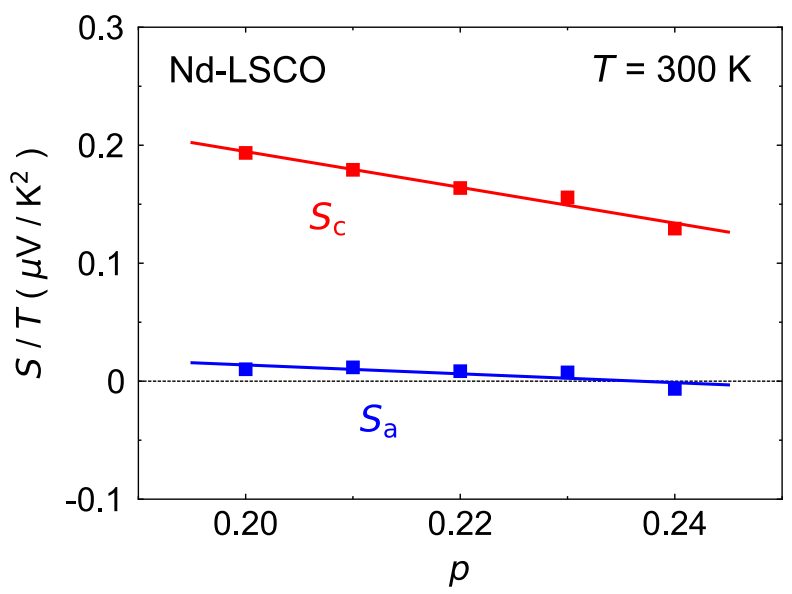

FIG. 6. $\quad S_{a} / T$ (blue) and $S_{c} / T$ (red) at $T=300 \mathrm{~K}$ (and $H=0$ ) as a function of the doping for Nd-LSCO $p=0.20,0.21,0.22$, 0.23 , and 0.24 . Solid lines are a linear fit to the data.
LSCO at $p=0.24$ obtained from ADMR [7,17] and ARPES measurements [15,27] (see the Appendix A for details). It is certainly not immediately obvious that the Boltzmann formalism can be successfully used to describe the non-Fermi-liquid regime considered in the present work. The justification of this procedure and a discussion of its limitations, including a comparison to a full Kubo calculation, are provided in Appendix D. We start by considering a constant scattering rate $1 / \tau$, independent of energy and momentum. In this case, the Seebeck coefficient does not depend on $\tau$, because it is a ratio of two transport coefficients- the Peltier coefficient $\alpha$ and the conductivity $\sigma$ - and it is given by the Mott formula at low temperature:

$S_{\mathrm{Mott}}=-\left.\frac{\pi^{2} k_{B}^{2} T}{3 e} \frac{\partial \ln \sigma_{i i}(\epsilon)}{\partial \epsilon}\right|_{\epsilon=0}=-\left.\frac{\pi^{2} k_{B}^{2} T}{3 e} \frac{\sigma_{i i}^{\prime}(\epsilon)}{\sigma_{i i}(\epsilon)}\right|_{\epsilon=0}$.

In this expression, $e$ is the electron charge, $k_{B}$ is the Boltzmann constant, $i=x, z$, and the Fermi level is set at $\epsilon=0 . \sigma(\epsilon)$ is the energy-dependent transport function which depends only on the band structure of the material
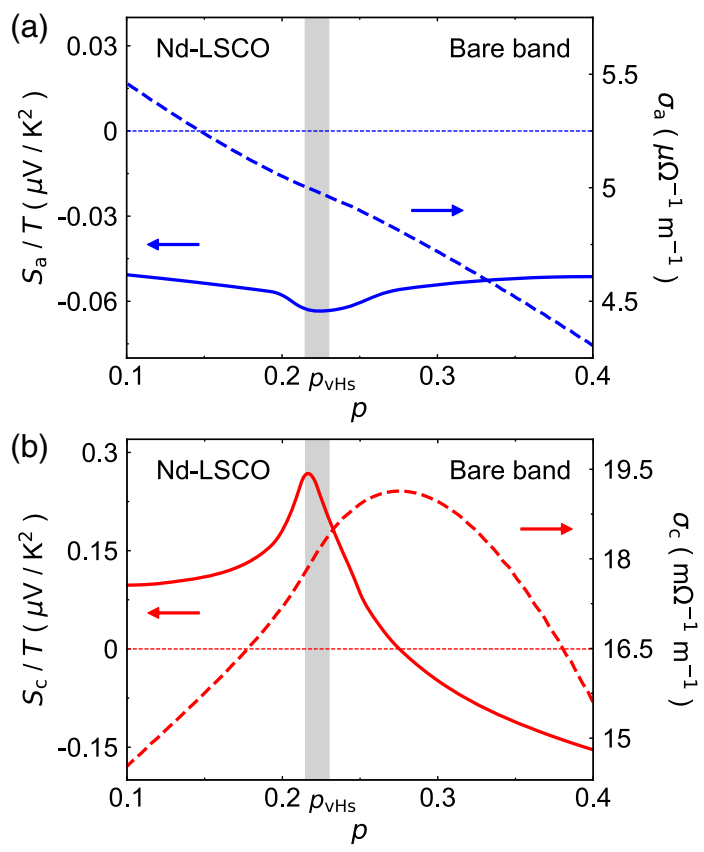

FIG. 7. Calculations of the Seebeck coefficient $(S / T$, full line, left axis) and conductivity ( $\sigma$, dashed line, right axis) as a function of the doping from the bare band dispersion of Nd-LSCO, as measured by ARPES [15,27], with an energy-independent scattering rate $1 / \tau$. (a) In-plane coefficients at $T=6 \mathrm{~K}$; (b) out-of-plane coefficients at $T=6 \mathrm{~K}$. We can see from the figures that the sign of $S / T$ is set by the slope of the conductivity $\sigma$ as a function of the doping. This reflects the Mott formula in Eq. (2). The minimum in $S_{a} / T$ and maximum in $S_{c} / T$ coincide approximately with the van Hove point ( $p_{\mathrm{vHs}}$, gray band) where the Fermi surface goes from holelike (below $p_{\mathrm{vHs}}$ ) to electronlike (above $p_{\mathrm{vHs}}$ ). 
for a constant $\tau$. Hence, the sign and magnitude of $S$ are entirely determined by the particle-hole asymmetry of the band structure in this case. In Fig. 7, we display the calculated $S$ for both directions as a function of the doping. These calculations are performed by allowing for the momentum dependence of the scattering rate along the Fermi surface, inferred from the ADMR measurements [17]. We see that the sign of $S$ is simply set by the slope of $\sigma(\epsilon=0)$ vs $p$ (the inverse of the resistivity $\rho$ ). In Fig. 7(a), the in-plane electrical conductivity $\sigma_{a}$ decreases monotonically with doping. This results in a negative $S_{a}$ predicted for all overdoped cuprates. The sign of $S_{a}$ does not change in the considered doping range, whereas the sign of $S_{c}$ flips (becomes negative) at high $p$. One can see that crossing the van Hove singularity around $p=p_{\mathrm{vHs}}$ has a large effect on the out-of-plane coefficient but a modest one for the inplane coefficient. This is because above $p_{\mathrm{vHs}}$ the Fermi surface loses states in the antinodal regions, where the $c$-axis dispersion is largest.

We turn to the temperature dependence of the Seebeck coefficients $S_{a} / T$ and $S_{c} / T$ in Nd-LSCO at $p=0.24$. Figure 8 shows a direct comparison between our experimental data (left column) and the calculations (right column). We see from Figs. 8(b) and 8(d) that the calculated $S(T)$ assuming an energy-independent (but momentum-dependent) scattering rate (dashed lines) disagrees with the data in magnitude for both directions. Furthermore, it yields the wrong sign for the in-plane component $S_{a}$, as also found in Ref. [12]. In summary,
Fig. 8 shows that neither the sign of $S_{a}$ nor the $T$ dependence and magnitude of $S_{a}$ and $S_{c}$ can be explained when assuming that particlelike and holelike excitations have equal scattering rates, even when taking into account the momentum dependence of this scattering rate along the Fermi surface.

We now consider the effect of a scattering rate which depends on energy, allowing for this energy dependence to reflect an asymmetry between particle and holes. The energy dependence of the inelastic scattering rate has long been recognized to be unconventional in cuprates. It is often described by the marginal Fermi liquid (MFL) form $[28,29]: 1 / \tau_{\mathrm{MFL}}=\sqrt{(a \epsilon)^{2}+\left[\alpha\left(k_{B} T\right) / \hbar\right]^{2}}$. As written, this expression is symmetric (even) under $\epsilon \rightarrow-\epsilon$, corresponding to equal scattering rates for particles and holes. Here, we introduce a modification of the MFL scattering rate with a particle-hole asymmetric energy dependence ["skewed" marginal Fermi liquid (SMFL)], namely,

$$
1 / \tau_{\mathrm{SMFL}}(\epsilon, T)=\sqrt{\left(a_{ \pm} \epsilon\right)^{2}+\left(\alpha \frac{k_{B} T}{\hbar}\right)^{2}}
$$

in which $a_{+}$applies to $\epsilon>0$ and $a_{-}$to $\epsilon<0$. The particlehole asymmetry is encoded in the difference between the coefficients $a_{+}$and $a_{-}$. We justify the use of the marginal Fermi liquid ansatz for Nd-LSCO at $p=0.24$ by the observation of perfectly $T$-linear resistivity (along both the $a$ axis and the $c$ axis) below $T \simeq 50 \mathrm{~K}[16,30]$. The
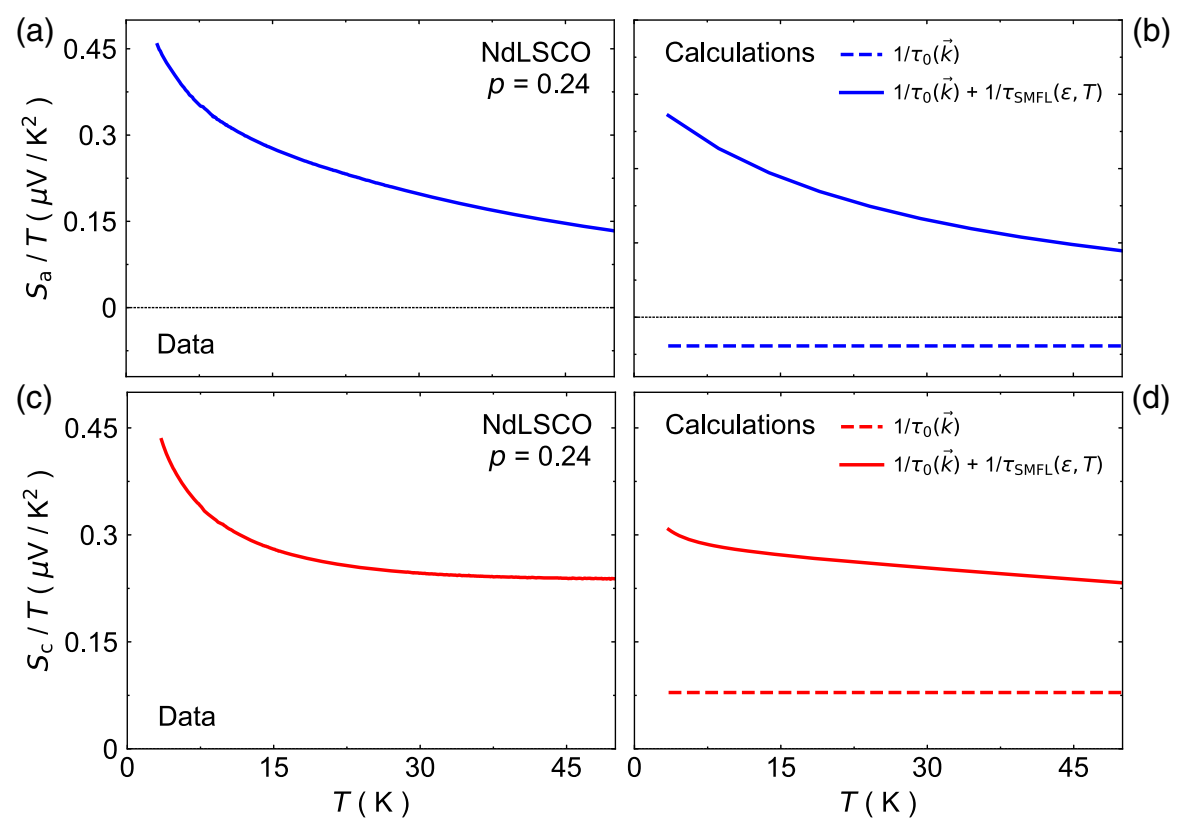

FIG. 8. Left: Seebeck coefficient of Nd-LSCO $p=0.24$ for both the $a$ axis (blue) (a) and $c$ axis (red) (c). Right: corresponding calculated Seebeck coefficients $(\mathrm{b}, \mathrm{d})$ for a momentum-dependent but energy-independent scattering $\operatorname{rate} 1 / \tau_{0}(\vec{k})=A+B|\cos (2 \phi)|^{\nu}$ (see Appendix A for more details) inferred from the elastic scattering rate extracted from ADMR measurements [17] (dashed line) and a momentum- and energy-dependent skewed scattering rate of the form $1 / \tau_{0}(\vec{k})+1 / \tau_{\mathrm{SMFL}}(\epsilon, T)$ (full line). 
dimensionless coefficient $\alpha=1.2 \pm 0.4$ extracted from ADMR [17] obeys the so-called Planckian limit [30,31]. In addition to this SMFL inelastic scattering rate, we also take into account, as above, the momentum-dependent elastic scattering rate $1 / \tau_{0}(\vec{k})$ obtained from the ADMR experiment [17] for Nd-LSCO at $p=0.24$. Hence, we use in our calculations the total scattering rate:

$$
1 / \tau(\epsilon, \vec{k}, T)=1 / \tau_{0}(\vec{k})+1 / \tau_{\mathrm{SMFL}}(\epsilon, T) .
$$

Note that the ADMR study yields the remarkable finding that the inelastic scattering rate is isotropic, i.e., $k$ independent. Correspondingly, we assume the energy dependence to also be isotropic; i.e., we take $a_{+}$and $a_{-}$to be $k$ independent. In Figs. 8(b) and 8(d), we see that the asymmetric scattering rate (plotted in Fig. 9 at $T=25 \mathrm{~K}$ ) changes the calculated $S(T)$ dramatically for both directions and matches well the experimental data. Crucially, the calculated $S_{a}(T)$ now has the correct sign. Furthermore, $S_{c}(T)$ is reproduced well, including the nontrivial plateau behavior between 15 and $60 \mathrm{~K}$. The same model and the exact same parameters lead to calculated in-plane and out-of-plane resistivities that also reproduces the data well in agreement with Ref. [17]. It is important to note that the particle-hole asymmetry of the scattering rate has a radical effect on the Seebeck coefficient but no effect on the resistivity.

In Fig. 10, we reveal the importance of incorporating the anisotropic elastic scattering rate $1 / \tau_{0}(\vec{k})$, inferred from the ADMR [17], in the calculations of the Seebeck coefficient. We see that using an isotropic scattering rate does not affect $S_{a}$ much; it greatly enhances the amplitude of $S_{c}$ and does not exhibit the experimentally observed plateau which is reproduced with an anisotropic scattering rate. It is not surprising that $S_{c}$ is more affected by a large anisotropic scattering rate, as most of the $c$-axis dispersion is located closer to the antinodal region of the Fermi surface, the same region whose contribution gets dramatically reduced by the anisotropy of the scattering rate. It therefore appears now that the plateau in $S_{c} / T$ below $60 \mathrm{~K}$ (Fig. 2) results from a competition between two effects that grow upon cooling: First, the skewness increases upon cooling (see Fig. 9), which increases $S_{c} / T$; second, the anisotropy of the scattering rate along the Fermi surface grows, which decreases $S_{c} / T$.

We now provide insight into the temperature dependence and sign of the Seebeck coefficient found from this calculation. The SMFL expression (3) is a particular example of a general class of skewed non-Fermi liquids recently discussed by two of us in Ref. [32]. In that work, it is shown that an inelastic scattering rate obeying an $\epsilon / T$ scaling with an intrinsic particle-hole asymmetry of the scaling function remarkably leads to a modification of the low-temperature value of the Seebeck coefficient, even in the presence of elastic scattering. This is in strong contrast

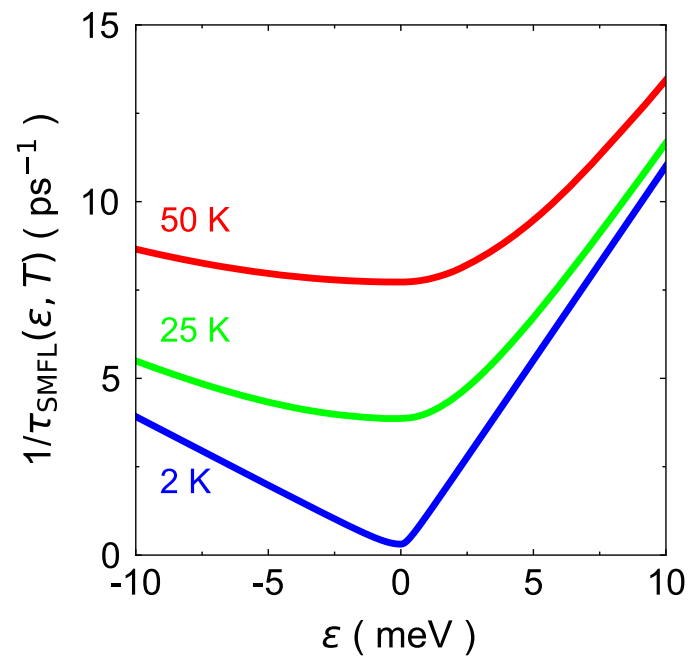

FIG. 9. Energy dependence of the skewed marginal Fermiliquid scattering rate $1 / \tau_{\mathrm{SMFL}}(\epsilon, T)$ at $T=2,25$, and $50 \mathrm{~K}$. $1 / \tau_{\mathrm{SMFL}}$ is defined by Eq. (3) with parameters given in Table II.

to Fermi liquids, in which the dominant term in the inelastic scattering rate is particle-hole-symmetric, and particlehole-asymmetric subdominant corrections affect the Seebeck coefficient only at high temperature but not in the low- $T$ regime dominated by elastic scattering [32,33]. In the case of the SMFL, following a simplification that is discussed in Appendix B and detailed in Ref. [32], in the $T \rightarrow 0$ limit we obtain

$$
\left.\frac{S}{T}\right|_{T=0} ^{\mathrm{SMFL}} \simeq \frac{1}{Z(T)}\left(\frac{S}{T}\right)_{\mathrm{Mott}}+c_{a} \frac{k_{B}^{2}}{e \hbar} \alpha \tau_{0} .
$$

In this expression, the Mott value corresponds to expression (2), which ignores the energy dependence of the scattering rate and whose sign is entirely determined by band structure. $Z(T)$ is a mass enhancement renormalization to which we return below. Here, we want to put the emphasis on the second term in Eq. (5), which involves a coefficient $c_{a}$ determined by the particle-hole asymmetry (skew) of the scattering rate $\left(c_{a}=\int_{0}^{\infty} d x x /\left[4 \cosh (x / 2)^{2}\right]\right.$ $\left[\sqrt{1+\left(a_{+} \hbar x / \alpha\right)^{2}}-\sqrt{1+\left(a_{-} \hbar x / \alpha\right)^{2}}\right] \approx 1.1 \hbar\left(a_{+}-a_{-}\right) / \alpha \approx$ 0.4 for the values of parameters reported in Appendix A), the dimensionless coupling constant $\alpha$ measuring the strength of the inelastic scattering, and the elastic scattering time $\tau_{0}$. This unconventional contribution to the Seebeck coefficient implies that the particle-hole asymmetry of the inelastic scattering rate can affect the magnitude and the sign of the Seebeck coefficient even in the low-temperature limit, where the magnitude of the inelastic scattering is small in comparison with the elastic scattering. This effect applies when the scattering rate is non-Fermi liquid and explains the observed sign change in the calculation above. We also note that, in contrast to the band (Mott) term, this contribution does not depend on any details of the band 


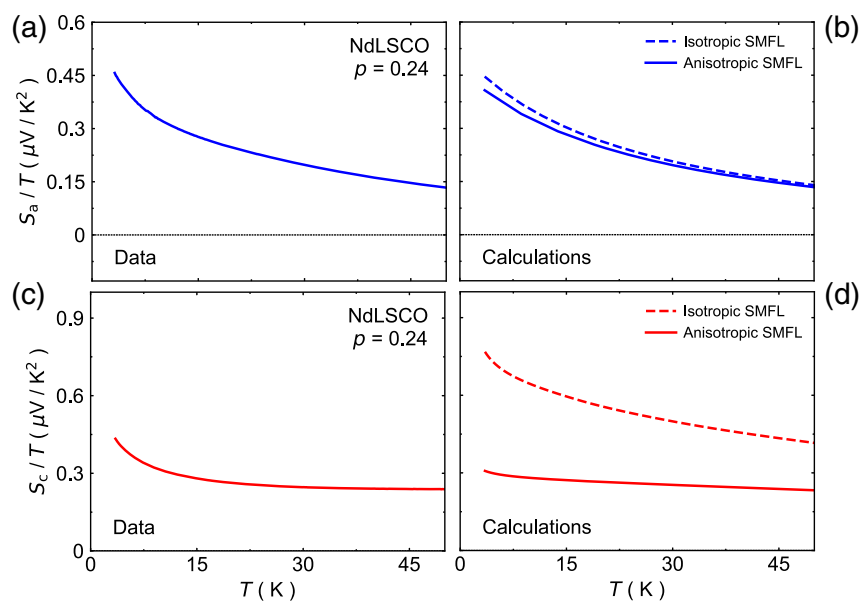

FIG. 10. Left: Seebeck coefficient of Nd-LSCO $p=0.24$ for both the $a$ axis (blue) (a) and $c$ axis (red) (c). Right: corresponding calculated Seebeck coefficients $(b, d)$ for a momentumindependent but energy-dependent skewed scattering rate $1 / \tau_{0}+1 / \tau_{\mathrm{SMFL}}(\epsilon, T)$, with $B=0$ in Eq. (A5) for the elastic scattering rate (isotropic, dashed line) and a momentum- and energy-dependent skewed scattering rate of the form $1 / \tau_{0}(\vec{k})+$ $1 / \tau_{\mathrm{SMFL}}(\epsilon, T)$ (anisotropic, full line), the same as in Fig. 8.

structure and is, in particular, isotropic. This may provide a hint into the observed isotropy of our experimental data at low temperature.

We now discuss the renormalization $Z(T)$. By KramersKronig, the ansatz for the scattering rate (imaginary part of the self-energy) with a linear energy or temperature dependence implies a logarithmic term in the low-energy slope of the real part of self-energy, that is, a mass renormalization $1 / Z(T)=m^{*} / m \propto[1+\log (\Lambda / T)]$, where $\Lambda$ is a cutoff. Indeed, a logarithmic enhancement of the specific heat coefficient is observed experimentally for Nd-LSCO at $p=$ 0.24 [8]. We see that this renormalization, in turn, implies a logarithmic behavior of $S / T$ at low temperatures, as seen in the first term in Eq. (5). It is important to notice, however, that this renormalization multiplies the band (Mott) part of the Seebeck coefficient. As a consequence, when the logarithmic mass term is included in our modeling, its effect is to increase $S_{c}$ (improving the agreement with the experiment) but also to diminish $S_{a}$ (worsening the agreement somewhat for this direction). A further understanding of the mass renormalization effect is left for future work. Note that logarithmic terms in the $T$ dependence of the Seebeck coefficient are discussed in Ref. [34] in relation to the proximity of a quantum critical point.

Recently, Jin et al. [35] reported measurements of the inplane Seebeck coefficient for LSCO $p=0.33$. At this doping, $S_{a}$ displays also a positive sign down to the lowest temperature, while it shows a $T^{2}$ resistivity characteristic of a Fermi liquid. Because particle-hole asymmetry in a Fermi liquid cannot cause a change of sign in the low-temperature regime [32], this could point to the influence of non-Fermi- liquid corrections at the antinodes even in the overdoped regime [36].

We conclude this section by contrasting our analysis with previous calculations of the Seebeck coefficient of other families of cuprate compounds for $p>p^{\star}$. In overdoped Bi2201 and Bi2212, in which the in-plane Seebeck coefficient is mostly negative, calculations by Kondo et al. [14] successfully reproduce the $T$ dependence of $S$ by using the electronic structure measured by ARPES and assuming that the scattering time depends on momentum in such a way as to maintain a constant scattering length $l=v \tau$, that is, $\tau(\vec{k}, \epsilon) \propto 1 / v(\vec{k}, \epsilon)$ (the same assumption is made in Ref. [37]). In this expression, $\vec{k}$ denotes a momentum on the Fermi surface and $\epsilon$ the energy of an excitation when moving away from the Fermi surface. The asymmetry between $\epsilon>0$ (particles) and $\epsilon<0$ (holes) in this model originates from the nearby van Hove singularity for momenta near the antinodes. Although this model is able to capture the temperature dependence and sign of the data, its validity is uncertain. Indeed, in this model, the scattering rate is the largest at the nodes, where the velocity is maximum. This is at odds with most of the literature on cuprates $[17,36,38,39]$.

Finally, it is interesting to note that, although quasi-1D organic superconductors can have very different physics from cuprates at the pseudogap critical point, calculations on a microscopic model show that incorporating the energy dependence of the scattering rate in those materials causes the Seebeck coefficient to change sign when close to the magnetic quantum critical point [40].

\section{B. $p<p^{\star}$ : Fermi surface transformation}

In Nd-LSCO, reducing the doping below $p^{\star}$ induces two main anomalies in the Seebeck coefficient at low temperatures: a large enhancement of $S_{a}$ and a negative $S_{c}$. Both are unambiguously connected with the onset of the pseudogap phase: They occur upon crossing $p^{\star}$ and at low temperatures below the pseudogap temperature $T^{\star}$. The sudden sign change in the out-of-plane Seebeck coefficient necessarily reflects a change in particle-hole asymmetry upon entering the pseudogap phase, either through a transformation of the electronic structure, and, therefore, the Fermi surface, or the scattering rate. As discussed previously [10], the enhancement in $S_{a}$ below $p^{\star}$ reflects the change in $n$ from $n \simeq 1+p$ above $p^{\star}$ to $n \simeq p$ below $p^{\star}$ associated with the Fermi surface transformation at $p^{\star}$ [7]. The origin of the negative $S_{c}$, however, is not clear and is the focus of the remaining discussion.

In Nd-LSCO, the Fermi surface undergoes two changes at $p^{\star}$. First, the large Fermi surface goes from holelike to electronlike when the van Hove singularity crosses the Fermi level, which occurs at a doping $p_{\mathrm{vHs}}$ that coincides with $p^{\star}$ [15]. As we show in Fig. 7, the bare band calculations predict no sign change in $S_{a}$ across $p_{\mathrm{vHs}}$ and a change to a positive sign in $S_{c}$, in contrast to our 
results. In Fig. 6, we plot $S_{a} / T$ and $S_{c} / T$ at $300 \mathrm{~K}$ as a function of the doping and see that both quantities decrease smoothly from $p=0.20$ to $p=0.24$, with no anomaly. Similarly, we observe that $S_{a} / T$ and $S_{c} / T$ show the same smooth increase upon cooling from $300 \mathrm{~K}$ down to about $100 \mathrm{~K}$ (Fig. 4), irrespective of doping. At those high temperatures above $T^{\star}$, only the vHs has an impact on the band structure, and, since there is no clear difference in the behaviors of $S_{a} / T$ and $S_{c} / T$, we conclude that the vHs alone is not the main cause for the negative $S_{c} / T$. Second, there is a transformation of the Fermi surface associated with the drop in carrier density as deduced from the Hall effect, resistivity [3], thermal conductivity [6], and in-plane Seebeck [10] measurements. This Fermi surface transformation was recently detected directly by ADMR measurements [7], which found that the Fermi surface below $p^{\star}$ is likely made of four nodal hole pockets, consistent with a transformation by a $Q=(\pi, \pi)$ wave vector. ARPES measurements see Fermi arcs that are consistent with the side of these pockets residing in the first Brillouin zone [15]. A model is needed to explain how such a Fermi surface transformation can account for a negative $S_{c}$.

The scattering rate, on the other hand, is not known to undergo a significant change at $p^{\star}$. Resistivity measurements on either side of $p^{\star}$ [3] find that the normal-state magnetoresistance (MR) is comparable at $p=0.22$ and $p=0.24$. In the weak-field limit, the magnetoresistance varies as $\mathrm{MR} \propto\left(\omega_{c} \tau\right)^{2}$, where $\omega_{c}$ is the cyclotron frequency and $\tau$ is the scattering time, suggesting that $\tau$ does not change significantly across $p^{\star}$. Similarly, in ADMR measurements on Nd-LSCO [7], the magnitude of the oscillations seen on each side of $p^{\star}$, at $p=0.21$ and $p=0.24$, is similar, implying that the amplitude of $\tau$ is roughly the same. So there is no indication, a priori, that the sign change in $S_{c}$ is correlated with a change in $\tau$ at the Fermi level. Finally, the fact that the change of $S_{a}$ through $p^{\star}$ roughly matches the ratio of the carrier densities further indicates that any change in $\tau$ is small.

A change in the Fermi surface, and specifically in the asymmetry of the band near the Fermi level, is, therefore, the likely cause of the negative $S_{c}$. It seems clear that without the pseudogap, which appears at temperatures below approximately $100 \mathrm{~K}$ over this doping range, $S_{a} / T$ and $S_{c} / T$ would keep evolving in tandem down to low temperatures, likely to match in the $T \rightarrow 0$ limit as they do at $p=0.24$. (This is actually shown to be the case by application of pressure to move $p^{\star}$ down below $p=0.22$ : The low- $T$ rise in $S_{a} / T$ at $p=0.22$ seen at ambient pressure is gone under pressure [41].) Consequently, we attribute the negative $S_{c}$ to a transformation of the band structure and, therefore, of the Fermi surface caused by the pseudogap phase. Based on ARPES [15] and recent ADMR measurements [7], the Fermi surface below $p^{\star}$ is truncated at the antinodes. Now this antinodal region is where $c$-axis dispersion is largest, thereby dominating, lending the bulk of the $c$-axis transport. From there, it is not hard to imagine that out-of-plane Seebeck would be most sensitive to a transformation of the Fermi surface in the $(\pi, 0)$ direction caused by the pseudogap phase. Further work is needed to model the sign change of $S_{c}$ below $p^{*}$.

\section{SUMMARY}

In summary, we have measured the Seebeck coefficient of the cuprate superconductor Nd-LSCO at dopings close to the critical doping $p^{\star}=0.23$ where the pseudogap phase ends at $T=0$. In particular, we examine the $c$-axis component $S_{c}$ for a heat current normal to the $\mathrm{CuO}_{2}$ planes, in the presence of a magnetic field sufficient to suppress superconductivity and, thus, track the normalstate behavior down to low temperature.

At $p>p^{\star}$, for $p=0.24$, we find that $S_{c}(T)$ and the inplane component $S_{a}(T)$ are both positive below $250 \mathrm{~K}$ and become roughly equal below $10 \mathrm{~K}$. This is in contrast to what calculations based on the well-characterized band structure predict, namely, that $S_{a}$ should be negative at all temperatures. We show that a good quantitative description of (and the correct sign for) both $S_{c}(T)$ and $S_{a}(T)$ is obtained if we add a linear asymmetric energy dependence to the scattering rate $1 / \tau$ previously extracted in Nd-LSCO as a function of the angle and temperature via ADMR measurements [17]. This suggests that $1 / \tau$ is Planckian not only in its $T$ dependence $\left(1 / \tau \simeq k_{B} T / \hbar\right)$, but also in its energy dependence $(1 / \tau \propto \epsilon)$, with an intrinsic particlehole asymmetry of the $\epsilon / T$ scaling function-as expected for a skewed Planckian metal [32].

At $p<p^{\star}$, we find that $S_{c}(T)$ undergoes a dramatic change at low temperature, suddenly becoming negative as soon as $p$ falls below $p^{\star}$. This is a striking new experimental signature of the pseudogap phase, which we attribute to a transformation of the Fermi surface. Further work is needed to identify what modification of the Fermi surface is responsible for this sign change.

\section{ACKNOWLEDGMENTS}

We thank K. Behnia, C. Bourbonnais, N. Hussey, and B. J. Ramshaw for stimulating discussions and Simon Fortier for his assistance with the experiments. L. T. acknowledges support from the Canadian Institute for Advanced Research (CIFAR) as a CIFAR Fellow and funding from the Institut Quantique, the Natural Sciences and Engineering Research Council of Canada (PIN:123817), the Fonds de Recherche du Québec-Nature et Technologies (FRQNT), the Canada Foundation for Innovation (CFI), and a Canada Research Chair. J. M. is supported by the Slovenian Research Agency (ARRS) under Program No. P1-0044 and Projects No. J1-2458, No. N1-0088, and No. J1-2455-1. J.-S. Z. was supported by NSF MRSEC under Cooperative Agreement No. DMR-1720595. This research was undertaken thanks in part to funding from the Canada First 
Research Excellence Fund and the Gordon and Betty Moore Foundation's EPiQS Initiative (Grant No. GBMF5306 to L. T.). The Flatiron Institute is a division of the Simons Foundation.

\section{APPENDIX A: SEEBECK CALCULATIONS}

The Seebeck coefficient is given by the ratio of the Peltier coefficient $\alpha_{i i}$ to the electrical conductivity $\sigma_{i i}$ (with $i=x, z), S_{i}=\alpha_{i i} / \sigma_{i i}$, where

$$
\begin{gathered}
\sigma_{i i}=\int_{-\infty}^{\infty} d \epsilon\left(-\frac{\partial f(\epsilon)}{\partial \epsilon}\right) \sigma_{i i}(\epsilon), \\
\alpha_{i i}=\int_{-\infty}^{\infty} d \epsilon\left[\left(-\frac{\partial f(\epsilon)}{\partial \epsilon}\right) \frac{\epsilon}{T}\right] \frac{\sigma_{i i}(\epsilon)}{-e}
\end{gathered}
$$

with $e$ the electron charge, $f(\epsilon)$ the Fermi-Dirac distribution, and

$$
\sigma_{i i}(\epsilon)=2 e^{2} \iiint_{\mathrm{BZ}} \frac{d^{3} k}{(2 \pi)^{3}} v_{i}(\vec{k})^{2} \tau(\vec{k}, \epsilon) \delta[\epsilon-E(\vec{k})],
$$

where $v_{i}(\vec{k})$ is the component of the quasiparticle velocity in the $i$ direction, $\tau(\vec{k}, \epsilon)$ is the quasiparticle lifetime depending on both momentum $\vec{k}$ and energy $\epsilon$, and $E(\vec{k})$ is the tight-binding band dispersion of Nd-LSCO [17,27].

In order to calculate Seebeck, we used the tight-binding model $E(\vec{k})$, measured by ADMR [17] and ARPES [27], to describe the band dispersion:

$$
\begin{aligned}
E(\vec{k})= & -2 t\left[\cos \left(k_{x} a\right)+\cos \left(k_{y} a\right)\right] \\
& -4 t^{\prime} \cos \left(k_{x} a\right) \cos \left(k_{y} a\right) \\
& -2 t^{\prime \prime}\left[\cos \left(2 k_{x} a\right)+\cos \left(2 k_{y} a\right)\right] \\
& -2 t_{z} \pi_{x} \pi_{y} \pi_{z}\left[\cos \left(k_{x} a\right)-\cos \left(k_{y} a\right)\right]^{2},
\end{aligned}
$$

with $a=3.75 \AA$ and $c=13.2 \AA$ the lattice constants, $\pi_{i}=$ $\cos \left(k_{i} a / 2\right)$ with $i=x, y$, and $\pi_{z}=\cos \left(k_{z} c / 2\right)$. The hopping parameters are found in Table I (from extended data Table I in Ref. [17]).

The angle-dependent elastic scattering rate is given by

$$
1 / \tau_{0}(\vec{k})=A+B|\cos (2 \phi)|^{\nu}
$$

This model is justified by the analysis of the ADMR data in Ref. [17]. Other functions with more parameters are also employed for comparison, and all end up with the same form factor for the elastic scattering rate (see the Methods section in Ref. [17] for more details).
TABLE I. Tight-binding parameters from the ADMR data of Nd-LSCO $p=0.24$ obtained from the angle-dependent magnetoresistance analysis in Ref. [17]. The hopping parameter $t=160 \mathrm{meV}$ is adjusted to meet the specific heat data on the same sample; more details can be found in the Methods section in Ref. [17].

\begin{tabular}{lccccc}
\hline \hline$t(\mathrm{meV})$ & $t^{\prime}$ & $t^{\prime \prime}$ & $t_{z}$ & $\mu$ & $p$ \\
\hline 160 & $-0.1364 t$ & $0.0682 t$ & $0.0651 t$ & $-0.8414 t$ & 0.259 \\
\hline \hline
\end{tabular}

TABLE II. Scattering rate parameters used in Eqs. (4) and (A5) to calculate the Seebeck coefficients in Fig. 8. The value of $\nu$ is derived from the angle-dependent magnetoresistance and is explained in more detail in the Methods section in Ref. [17].

\begin{tabular}{lccccc}
\hline \hline$a_{-}\left(\mathrm{ps}^{-1} / \mathrm{meV}\right)$ & $a_{+}\left(\mathrm{ps}^{-1} / \mathrm{meV}\right)$ & $\alpha$ & $A\left(\mathrm{ps}^{-1}\right)$ & $B\left(\mathrm{ps}^{-1}\right)$ & $\nu$ \\
\hline 0.4 & 1.1 & 1.2 & 9.97 & 71.1 & 12 \\
\hline \hline
\end{tabular}

\section{APPENDIX B: COMPARISON BETWEEN KUBO AND BOLTZMANN RESULTS}

The use of a Boltzmann formalism in the relaxation time approximation to describe non-Fermi-liquid regimes deserves further discussion and justification, which is the subject of this section. First, it is important to realize that one can derive Boltzmann-like expressions as given by Eqs. (A1)-(A3) starting from the Kubo formula, without assuming the scattering rate to be of the Fermi-liquid form $[32,42,43]$. We neglect vertex corrections (we leave investigation of effects of those for future work) and assume for simplicity a single electronic band, but otherwise keep the discussion general. The Kubo expression for the transport coefficients $L_{n}$ is

$$
L_{n}=(2 \pi) \int d \omega \omega^{n}\left(-\frac{d f}{d \omega}\right) \sum_{\vec{k}}\left(v_{0 \vec{k}}^{\alpha}\right)^{2} A_{\vec{k}}^{2} .
$$

Note that, in contrast to the Boltzmann formalism, this expression involves the bare (band structure) velocity denoted by $v_{0 \vec{k}}^{\alpha}$ and the full electronic spectral function. The latter is given by

$$
A_{\vec{k}}(\omega)=-\frac{1}{\pi} \operatorname{Im} \frac{1}{\omega-\varepsilon_{\vec{k}}-\Sigma_{\vec{k}}(\omega)}
$$

with $\varepsilon_{\vec{k}}$ the band energy (shifted by the chemical potential) and $\Sigma_{\vec{k}}(\omega)$ the self-energy of an electron with momentum $\vec{k}$ and excitation energy $\omega$. This expression simplifies to the Boltzmann-like formalism when $A_{\vec{k}}(\omega)^{2}$ can be approximated as $Z^{2} \delta\left(\omega-\omega_{\vec{k}}\right) \tau_{\vec{k}} / \pi$, in which $\omega_{\vec{k}}$ is the quasiparticle energy at which the spectral function is peaked, given by the solution of $\omega-\varepsilon_{\vec{k}}-\operatorname{Re} \Sigma_{\vec{k}}(\omega)=0 . Z$ is the spectral 
weight carried by this peak, and $1 / \tau_{\vec{k}}=-2 Z \operatorname{Im} \Sigma_{\vec{k}}\left(\omega_{\vec{k}}\right)$ is the inverse quasiparticle transport lifetime. Introducing the renormalized quasiparticle velocity $v_{\vec{k}}^{\alpha}=Z v_{0 \vec{k}}^{\alpha}$, one finally obtains the Boltzmann form:

$$
L_{n}=\left.2 \int \frac{d^{3} k}{(2 \pi)^{3}} \omega_{\vec{k}}^{n}\left(-\frac{d f}{d \omega}\right)\right|_{\omega=\omega_{\vec{k}}}\left(v_{\vec{k}}^{\alpha}\right)^{2} \tau_{\vec{k}} .
$$

In a Fermi liquid, quasiparticle excitations dispersing as $\omega_{\vec{k}}$ have a very long lifetime $\tau_{\vec{k}} \sim 1 / T^{2}$ at low $T$ and a finite $Z$; hence, the quasiparticle peak is very narrow, and the above replacement leading to the Boltzmann approximation is justified. However, Landau Fermi-liquid behavior is not a necessary condition, and the Boltzmann approximation can apply even under milder conditions. In Ref. [43], it is shown, for example, that a sufficient condition is that the peak in the spectral function is narrower than $T$. We also note that in a non-Fermi liquid $Z$ remains nonzero at finite $T$, even when it vanishes asymptotically as $T \rightarrow 0$. An alternative derivation is given in Refs. [32,42], which assume, however, a momentum-independent scattering rate. However, the justification given in these articles does not apply in the present case, since we include impurity scattering which dominates at low $T$ (hence, the spectral function peak is not narrower than $T$ ) and, furthermore, this scattering is momentum dependent. Hence, we must proceed differently to justify the Boltzmann approximation.

\section{Derivation of Boltzmann transport from Kubo formula for momentum-dependent scattering}

We assume the transport to be metallic, i.e., dominated by contributions close to the Fermi surface (this means that transport is associated with electrons within a sufficiently thin shell in momentum space but does not imply Fermiliquid properties of the corresponding states). We reformulate the momentum integral in Eq. (B1) by introducing equienergy $\varepsilon_{\vec{k}}+\operatorname{Re} \Sigma_{\vec{k}}=$ const $=\omega$ surfaces in momentum space. We introduce a new set of coordinates $S_{k}, k_{\perp}$, where $k_{\perp}$ denotes the distance from the equienergy surface at $\omega$ and $S_{k}$ denotes the position on that surface.

That is, we write

$$
L_{n}=2 \pi \int d S_{k} \int d \omega \omega^{n}\left(-\frac{d f}{d \omega}\right) \int d k\left(v_{0 k_{\perp}, S_{k}}^{\alpha}\right)^{2} A_{k_{\perp}, S_{k}}^{2} J
$$

where $J=J\left(k_{\perp}, S_{k}\right)$ is the Jacobian. At each position at the Fermi surface $S_{k}$ and for each $\omega$, the spectral functions peak at a perpendicular momentum $k_{\perp}=k_{\omega}$ given by solution of the equation $\omega-\varepsilon_{k_{\perp}, S_{k}}-\operatorname{Re} \Sigma_{k_{\perp}, S_{k}}(\omega)=0$. Linearizing in $k_{\perp}$ around $k_{\omega}$, introducing the corresponding "Fermi velocity"

$$
\tilde{v}=\left.\left[\partial\left(\varepsilon_{k_{\perp}, S_{k}}+\Sigma_{k_{\perp}, S_{k}}\right) / \partial k_{\perp}\right]\right|_{k_{\perp}=k_{\omega}},
$$

and approximating the values of the band velocities and the Jacobian in the integral over $k_{\perp}$ in Eq. (B4) by the value at $k_{\omega}$ the integrand, there becomes the square of Lorentzian function. Extending the integral over $k_{\perp}$ to $(-\infty, \infty)$ and using $\int d x /\left(1+x^{2}\right)^{2}=\pi / 2$, one gets

$L_{n}=2 \int d S_{k} \int d \omega \omega^{n}\left(-\frac{d f}{d \omega}\right) \frac{\left(v_{0 k_{\omega}, S_{k}}^{\alpha}\right)^{2}}{\tilde{v}\left(-2 \operatorname{Im} \Sigma_{k_{\omega}, S_{k}}\right)} J$.

One can, by reintroducing a momentum variable $k_{\perp}$ set by relation $\omega=\omega\left(k_{\perp}\right)$ and using $d \omega=\tilde{v} d k_{\perp}+$ $[\partial \operatorname{Re} \Sigma(\omega) / \partial \omega] d \omega$ and $Z=1-\partial \operatorname{Re} \Sigma / \partial \omega$, rewrite this expression to

$$
L_{n}=\left.2 \int \frac{d^{3} k}{(2 \pi)^{3}} \omega_{\vec{k}}^{n}\left(-\frac{d f}{d \omega}\right)\right|_{\omega=\omega_{\vec{k}}}\left(v_{\vec{k}}^{\alpha}\right)^{2} \tau_{\vec{k}},
$$

which is the Boltzmann form Eq. (B3). Notice that quasiparticle velocities and lifetimes appear in this expression.

Approximating $J,\left(v_{k, S_{k}}^{\alpha}\right)^{2}$, and $\operatorname{Im} \Sigma_{k, S_{k}}$ by the value at $k_{\omega}$ and taking them out of the $k_{\perp}$ integral in Eq. (B4) is valid when these quantities vary slowly in $k$, a condition that can be specified in terms of

$$
\frac{1}{v_{F}}\left(\frac{d \log \zeta}{d k_{\perp}}\right)(-\operatorname{Im} \Sigma) \ll 1
$$

for quantities $\zeta=J, v_{k_{\perp}, S_{k}}^{\alpha},-\operatorname{Im} \Sigma_{k_{\perp}, S_{k}}$, and that needs to hold for all $S_{k}$. Crucial for the discussion that follows is the appearance of the Fermi velocity $v_{\omega, S_{k}} \sim v_{F}$. When this is small (such as close to van Hove singularities), this condition becomes more difficult to satisfy. Likewise, close to the boundaries of the Brillouin zone, also extending the boundaries of momentum integral becomes problematic.

\section{Numerical verification}

For a more complete confirmation of the validity of the Boltzmann approach in the present case, hence, we investigated the issue numerically. In order to set up a Kubo calculation, we construct a self-energy with the imaginary part given by (half of) the scattering rate extracted from ADMR experiments and used in the Boltzmann calculations. Because the renormalizations are already included in the extracted tight-binding parameters, we neglect the real part of self-energy. The results are shown in Fig. 11. One sees that the Boltzmann description captures the results obtained using the Kubo formula quite well. In particular, for the in-plane response, the calculated Seebeck coefficient lies on top of the Boltzmann description. For the outof-plane, the deviations are larger but remain $\lesssim 20 \%$, which shows that there is no qualitative breakdown of the 

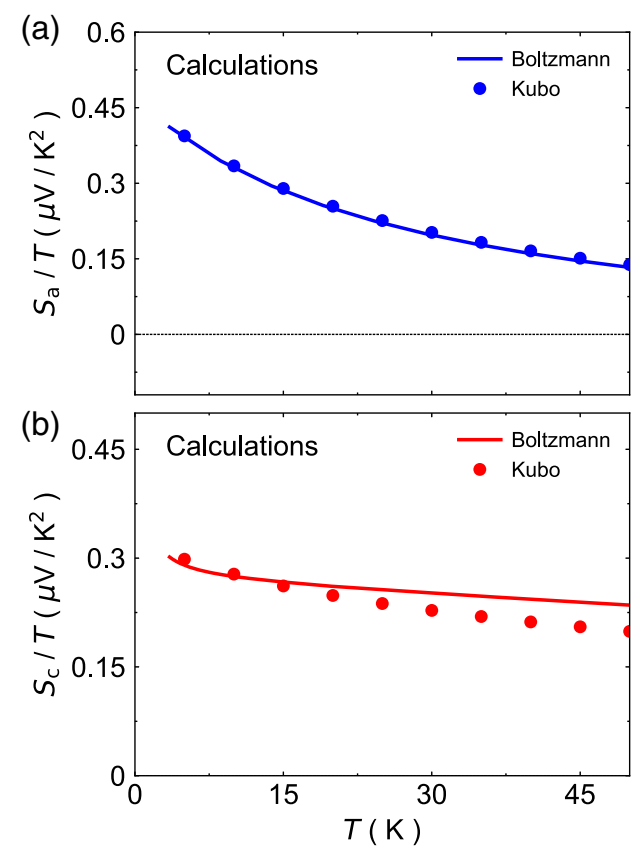

FIG. 11. Comparison between the Kubo and Boltzmann evaluations of the Seebeck coefficient: (a) in-plane $S_{a}$; (b) out-ofplane $S_{c}$.

Boltzmann description even though the energy and temperature dependence of the considered scattering are nonFermi liquid and strongly angular dependent. This is a direct demonstration of the validity of the Boltzmann-like description used in the main text.

Why are the deviations in the in-plane case smaller? To understand this, it is worth recalling that the band velocities in the $x$ direction are very small at the antinodes, whereas they are largest there in the $z$ direction. This is shown also on the contour map in Figs. 12(a) and 12(b) that depicts $\left(v_{\vec{k}}^{\alpha}\right)^{2}$ for the in-plane $(\alpha=x)$ and out-of-plane $(\alpha=z)$ cases, respectively.

That is, in the in-plane case, the band transport function suppresses the contribution from the antinodes. There, the Fermi velocities are smaller and the condition in Eq. (B7) is satisfied to a lesser degree.

One can rationalize this also from another point of view, because the transport function retains in the in-plane case just momenta at the nodes means that one can treat the scattering as momentum independent, with the magnitude given by the value at the nodes. Hence, for the in-plane case, one can apply the derivation of the Boltzmann transport for the case without momentum dependence [32], whereas this cannot be done for the out-of-plane case. One can illustrate this qualitative distinction also by inspecting $\left(v_{\vec{k}}^{\alpha}\right)^{2} \tau_{\vec{k}}$ that is shown in Figs. 12(c) and 12(d) for the in-plane (out-of-plane) case, respectively. For the inplane case, Fig. 12(c) resembles Fig. 12(a), which tells that momentum dependence of scattering is not important. For the out-of-plane response, this is not the case: The (a)
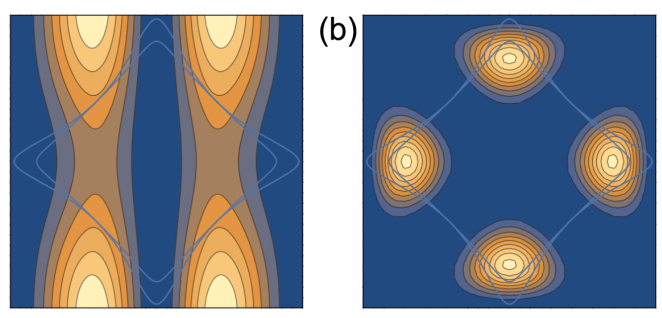

(c)
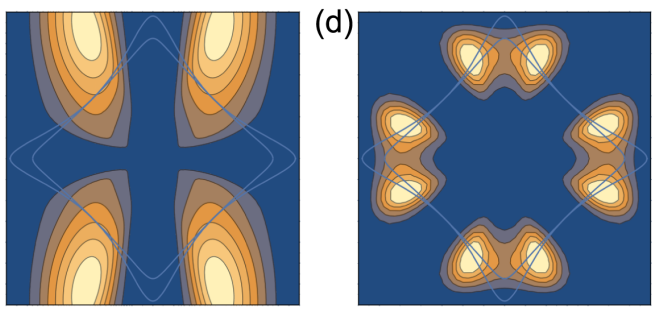

FIG. 12. Color maps of (a) $v_{x}^{2}$, (b) $v_{z}^{2}$, (c) $v_{x}^{2} \tau$, and $v_{z}^{2} \tau$ in the Brillouin zone. The Fermi surfaces at $k_{z}=0, \pi$ are also shown. The lifetime $\tau$ is evaluated at $T \rightarrow 0$; the data for (a),(c) and (b), (d) are evaluated for $k_{z}=0$ and $\pi / 2$, respectively.

velocities are largest where the scattering is also large and the interplay between the two must be taken into account.

[1] C. Proust and L. Taillefer, The Remarkable Underlying Ground States of Cuprate Superconductors, Annu. Rev. Condens. Matter Phys. 10, 409 (2019).

[2] S. Badoux, W. Tabis, F. Laliberté, G. Grissonnanche, B. Vignolle, D. Vignolles, J. Béard, D. A. Bonn, W. N. Hardy, R. Liang, N. Doiron-Leyraud, L. Taillefer, and C. Proust, Change of Carrier Density at the Pseudogap Critical Point of a Cuprate Superconductor, Nature (London) 531, 210 (2016).

[3] C. Collignon, S. Badoux, S. A. A. Afshar, B. Michon, F. Laliberté, O. Cyr-Choinière, J.-S. Zhou, S. Licciardello, S. Wiedmann, N. Doiron-Leyraud, and L. Taillefer, FermiSurface Transformation across the Pseudogap Critical Point of the Cuprate Superconductor $\mathrm{La}_{1.6-x} \mathrm{Nd}_{0.4} \mathrm{Sr}_{x} \mathrm{CuO}_{4}$, Phys. Rev. B 95, 224517 (2017).

[4] M. Lizaire, A. Legros, A. Gourgout, S. Benhabib, S. Badoux, F. Laliberté, M.-E. Boulanger, A. Ataei, G. Grissonnanche, D. LeBoeuf, S. Licciardello, S. Wiedmann, S. Ono, H. Raffy, S. Kawasaki, G.-Q. Zheng, N. DoironLeyraud, C. Proust, and L. Taillefer, Transport Signatures of the Pseudogap Critical Point in the Cuprate Superconductor $\mathrm{Bi}_{2} \mathrm{Sr}_{2-x} \mathrm{La}_{x} \mathrm{CuO}_{6+\delta}$, Phys. Rev. B 104, 014515 (2021).

[5] F. Laliberté, W. Tabis, S. Badoux, B. Vignolle, D. Destraz, N. Momono, T. Kurosawa, K. Yamada, H. Takagi, N. Doiron-Leyraud, C. Proust, and L. Taillefer, Origin of the Metal-to-Insulator Crossover in Cuprate Superconductors, arXiv:1606.04491.

[6] B. Michon, A. Ataei, P. Bourgeois-Hope, C. Collignon, S. Y. Li, S. Badoux, A. Gourgout, F. Laliberté, J.-S. Zhou, N. Doiron-Leyraud, and L. Taillefer, Wiedemann-Franz. 
Law and Abrupt Change in Conductivity across the Pseudogap Critical Point of a Cuprate Superconductor, Phys. Rev. X 8, 041010 (2018).

[7] Y. Fang, G. Grissonnanche, A. Legros, S. Verret, F. Laliberté, C. Collignon, A. Ataei, M. Dion, J. Zhou, D. Graf, M. J. Lawler, P. Goddard, L. Taillefer, and B. J. Ramshaw, Fermi Surface Transformation at the Pseudogap Critical Point of a Cuprate Superconductor, arXiv: 2004.01725.

[8] B. Michon, C. Girod, S. Badoux, J. Kačmarčík, Q. Ma, M. Dragomir, H. A. Dabkowska, B. D. Gaulin, J.-S. Zhou, S. Pyon, T. Takayama, H. Takagi, S. Verret, N. Doiron-Leyraud, C. Marcenat, L. Taillefer, and T. Klein, Thermodynamic Signatures of Quantum Criticality in Cuprate Superconductors, Nature (London) 567, 218 (2019).

[9] C. Girod, D. LeBoeuf, A. Demuer, G. Seyfarth, S. Imajo, K. Kindo, Y. Kohama, M. Lizaire, A. Legros, A. Gourgout, H. Takagi, T. Kurosawa, M. Oda, N. Momono, J. Chang, S. Ono, G.-q. Zheng, C. Marcenat, L. Taillefer, and T. Klein, Normal State Specific Heat in the Cuprate Superconductors $\mathrm{La}_{2-x} \mathrm{Sr}_{x} \mathrm{CuO}_{4}$ and $\mathrm{Bi}_{2+y} \mathrm{Sr}_{2-x-y} \mathrm{La}_{x} \mathrm{CuO}_{6+\delta}$ near the Critical Point of the Pseudogap Phase, Phys. Rev. B 103, 214506 (2021).

[10] C. Collignon, A. Ataei, A. Gourgout, S. Badoux, M. Lizaire, A. Legros, S. Licciardello, S. Wiedmann, J.-Q. Yan, J.-S. Zhou, Q. Ma, B. D. Gaulin, N. Doiron-Leyraud, and L. Taillefer, Thermopower across the Phase Diagram of the Cuprate $\mathrm{La}_{1.6-x} \mathrm{Nd}_{0.4} \mathrm{Sr}_{x} \mathrm{CuO}_{4}$ : Signatures of the Pseudogap and Charge-Density-Wave Phases, Phys. Rev. B 103, 155102 (2021).

[11] K. Behnia, D. Jaccard, and J. Flouquet, On the Thermoelectricity of Correlated Electrons in the Zero-Temperature Limit, J. Phys. Condens. Matter 16, 5187 (2004).

[12] S. Verret, O. Simard, M. Charlebois, D. Sénéchal, and A.-M. S. Tremblay, Phenomenological Theories of the LowTemperature Pseudogap: Hall Number, Specific Heat, and Seebeck Coefficient, Phys. Rev. B 96, 125139 (2017).

[13] K. Behnia, Fundamentals of Thermoelectricity, Illustrated Edition (Oxford University Press, 2015), 10.1093/acprof: oso/9780199697663.001.0001.

[14] T. Kondo, T. Takeuchi, U. Mizutani, T. Yokoya, S. Tsuda, and S. Shin, Contribution of Electronic Structure to Thermoelectric Power in $\mathrm{BiPb}_{2} \mathrm{SrLa}_{2} \mathrm{CuO}_{6+\delta}$, Phys. Rev. B 72, 024533 (2005).

[15] C. E. Matt et al., Electron Scattering, Charge Order, and Pseudogap Physics in $\mathrm{La}_{1.6-x} \mathrm{Nd}_{0.4} \mathrm{Sr}_{x} \mathrm{CuO}_{4}$ : An AngleResolved Photoemission Spectroscopy Study, Phys. Rev. B 92, 134524 (2015).

[16] R. Daou, N. Doiron-Leyraud, D. LeBoeuf, S. Y. Li, F. Laliberté, O. Cyr-Choinière, Y. J. Jo, L. Balicas, J.-Q. Yan, J.-S. Zhou, J. B. Goodenough, and L. Taillefer, Linear Temperature Dependence of Resistivity and Change in the Fermi Surface at the Pseudogap Critical Point of a High- $T_{c}$ Superconductor, Nat. Phys. 5, 31 (2009).

[17] G. Grissonnanche, Y. Fang, A. Legros, S. Verret, F. Laliberté, C. Collignon, J. Zhou, D. Graf, P. A. Goddard, L. Taillefer, and B.J. Ramshaw, Linear-in Temperature Resistivity from an Isotropic Planckian Scattering Rate, Nature (London) 595, 667 (2021).
[18] H. Wang, F. Yang, Y. Guo, K. Peng, D. Wang, W. Chu, and $\mathrm{S}$. Zheng, Determination of the Thermopower of Microscale Samples with an ac Method, Measurement 131, 204 (2019).

[19] R. Daou, O. Cyr-Choinière, F. Laliberté, D. LeBoeuf, N. Doiron-Leyraud, J.-Q. Yan, J.-S. Zhou, J. B. Goodenough, and L. Taillefer, Thermopower across the Stripe Critical Point of $\mathrm{La}_{1.6-x} \mathrm{Nd}_{0.4} \mathrm{Sr}_{x} \mathrm{CuO}_{4}$ : Evidence for a Quantum Critical Point in a Hole-Doped High- $T_{c}$ Superconductor, Phys. Rev. B 79, 180505(R) (2009).

[20] F. Laliberté, J. Chang, N. Doiron-Leyraud, E. Hassinger, R. Daou, M. Rondeau, B. Ramshaw, R. Liang, D. Bonn, W. Hardy, S. Pyon, T. Takayama, H. Takagi, I. Sheikin, L. Malone, C. Proust, K. Behnia, and L. Taillefer, FermiSurface Reconstruction by Stripe Order in Cuprate Superconductors, Nat. Commun. 2, 432 (2011).

[21] G. Grissonnanche, A. Legros, S. Badoux, E. Lefrançois, V. Zatko, M. Lizaire, F. Laliberté, A. Gourgout, J.-S. Zhou, S. Pyon, T. Takayama, H. Takagi, S. Ono, N. Doiron-Leyraud, and L. Taillefer, Giant Thermal Hall Conductivity in the Pseudogap Phase of Cuprate Superconductors, Nature (London) 571, 376 (2019).

[22] G. Grissonnanche, S. Thériault, A. Gourgout, M.-E. Boulanger, E. Lefrançois, A. Ataei, F. Laliberté, M. Dion, J.-S. Zhou, S. Pyon, T. Takayama, H. Takagi, N. Doiron-Leyraud, and L. Taillefer, Chiral Phonons in the Pseudogap Phase of Cuprates, Nat. Phys. 16, 1108 (2020).

[23] S. D. Obertelli, J. R. Cooper, and J. L. Tallon, Systematics in the Thermoelectric Power of High- $T_{c}$ Oxides, Phys. Rev. B 46, 14928 (1992).

[24] F. Munakata, K. Matsuura, K. Kubo, T. Kawano, and $\mathrm{H}$. Yamauchi, Thermoelectric Power of $\mathrm{Bi}_{2} \mathrm{Sr}_{2} \mathrm{Ca}_{1-x} \mathrm{Y}_{x} \mathrm{Cu}_{2} \mathrm{O}_{8+y}$, Phys. Rev. B 45, 10604 (1992).

[25] M. V. Elizarova and V. E. Gasumyants, Band Spectrum Transformation and $T_{c}$ Variation in the $\mathrm{La}_{2-x} \mathrm{Sr}_{x} \mathrm{CuO}_{y}$ System in the Underdoped and Overdoped Regimes, Phys. Rev. B 62, 5989 (2000).

[26] A. Yamamoto, W.-Z. Hu, and S. Tajima, Thermoelectric Power and Resistivity of $\mathrm{HgBa}_{2} \mathrm{CuO}_{4+\delta}$ over a Wide Doping Range, Phys. Rev. B 63, 024504 (2000).

[27] M. Horio et al., Three-Dimensional Fermi Surface of Overdoped La-Based Cuprates, Phys. Rev. Lett. 121, 077004 (2018).

[28] C. M. Varma, P. B. Littlewood, S. Schmitt-Rink, E. Abrahams, and A. E. Ruckenstein, Phenomenology of the Normal State of Cu-O High-Temperature Superconductors, Phys. Rev. Lett. 63, 1996 (1989).

[29] C. M. Varma, Colloquium: Linear in Temperature Resistivity and Associated Mysteries including High Temperature Superconductivity, Rev. Mod. Phys. 92, 031001 (2020).

[30] A. Legros, S. Benhabib, W. Tabis, F. Laliberté, M. Dion, M. Lizaire, B. Vignolle, D. Vignolles, H. Raffy, Z. Z. Li, P. Auban-Senzier, N. Doiron-Leyraud, P. Fournier, D. Colson, L. Taillefer, and C. Proust, Universal T-Linear Resistivity and Planckian Dissipation in Overdoped Cuprates, Nat. Phys. 15, 142 (2019).

[31] J. A. N. Bruin, H. Sakai, R. S. Perry, and A. P. Mackenzie, Similarity of Scattering Rates in Metals Showing T-Linear Resistivity, Science 339, 804 (2013). 
[32] A. Georges and J. Mravlje, Skewed Non-Fermi Liquids and the Seebeck Effect, Phys. Rev. Research 3, 043132 (2021).

[33] K. Haule and G. Kotliar, in Properties and Applications of Thermoelectric Materials, edited by V. Zlatic and A.C. Hewson (Springer, New York, 2009), pp. 119-131.

[34] I. Paul and G. Kotliar, Thermoelectric Behavior near the Magnetic Quantum Critical Point, Phys. Rev. B 64, 184414 (2001).

[35] H. Jin, A. Narduzzo, M. Nohara, H. Takagi, N. E. Hussey, and K. Behnia, Positive Seebeck Coefficient in Highly Doped $\mathrm{La}_{2-x} \mathrm{Sr}_{x} \mathrm{CuO}_{4}(x=0.33)$; Its Origin and Implication, J. Phys. Soc. Jpn. 90, 053702 (2021).

[36] J. Chang, M. Månsson, S. Pailhès, T. Claesson, O. J. Lipscombe, S. M. Hayden, L. Patthey, O. Tjernberg, and J. Mesot, Anisotropic Breakdown of Fermi Liquid Quasiparticle Excitations in Overdoped $\mathrm{La}_{2-x} \mathrm{Sr}_{x} \mathrm{CuO}_{4}$, Nat. Commun. 4, 2559 (2013).

[37] J. G. Storey, J. L. Tallon, and G. V. M. Williams, Electron Pockets and Pseudogap Asymmetry Observed in the Thermopower of Underdoped Cuprates, Europhys. Lett. 102, 37006 (2013).

[38] M. Abdel-Jawad, M. P. Kennett, L. Balicas, A. Carrington, A. P. Mackenzie, R. H. McKenzie, and N.E. Hussey, Anisotropic Scattering and Anomalous Normal-State
Transport in a High-Temperature Superconductor, Nat. Phys. 2, 821 (2006).

[39] T. Yoshida, X. J. Zhou, D. H. Lu, S. Komiya, Y. Ando, H. Eisaki, T. Kakeshita, S. Uchida, Z. Hussain, Z.-X. Shen, and A. Fujimori, Low-Energy Electronic Structure of the High- $T_{c}$ Cuprates $\mathrm{La}_{2-x} \mathrm{Sr}_{x} \mathrm{CuO}_{4}$ Studied by Angle-Resolved Photoemission Spectroscopy, J. Phys. Condens. Matter 19, 125209 (2007).

[40] M. Shahbazi and C. Bourbonnais, Seebeck Coefficient in Correlated Low-Dimensional Organic Metals, Phys. Rev. B 94, 195153 (2016).

[41] A. Gourgout, A. Ataei, M.-E. Boulanger, S. Badoux, S. Thériault, D. Graf, J.-S. Zhou, S. Pyon, T. Takayama, H. Takagi, N. Doiron-Leyraud, and L. Taillefer, Effect of Pressure on the Pseudogap and Charge Density Wave Phases of the Cuprate Nd-LSCO Probed by Thermopower Measurements, Phys. Rev. Research 3, 023066 (2021).

[42] X. Deng, J. Mravlje, R. Žitko, M. Ferrero, G. Kotliar, and A. Georges, How Bad Metals Turn Good: Spectroscopic Signatures of Resilient Quasiparticles, Phys. Rev. Lett. 110, 086401 (2013).

[43] W. Xu, K. Haule, and G. Kotliar, Hidden Fermi Liquid, Scattering Rate Saturation, and Nernst Effect: A Dynamical Mean-Field Theory Perspective, Phys. Rev. Lett. 111, 036401 (2013). 